\title{
Multi-objective Genetic Algorithm based on Game Theory and its Application
}

\author{
Jian Chi ${ }^{1, \text { a }}$, Yanfei Liu ${ }^{2, b}$ \\ ${ }^{1}$ Hebei Normal University for Nationality , Department of Mathematics and Computer \\ Chengde,China \\ ${ }^{2}$ Hebei Normal University for Nationality, Department of Mathematics and Computer \\ Chengde,China \\ aleekchj@gmail.com, ${ }^{b}$ merrylyf@gmail.com
}

Keywords: Multi-objective genetic algorithm, Game theory, application

\begin{abstract}
Mufti-objective optimization has been a difficult problem and focus for research in fields of science and engineering. There already have a lot of classical methods for solving mufti-objective optimization problems before evolutionary algorithms were introduced in 1985. Classical muftiobjective optimization methods have been thoroughly developed, but there are still Lots of shortcomings in solving high dimension, multimodal problems. GAs can handle large space of problem and get a lot of trade-of fronts (possible solutions) in one evolution. A GA does not need much information about the problem before starting the optimization process, also it is not sensitive to the convex of the defined fields of the objective functions. So using GAs in solving mufti-objective optimization problems is the most important research direction in the future. We import knowledge of immune, co-evolution and game theory into genetic algorithm to improve the performance on solving the mufti-objective optimization problems. The results of the riments show that all of them can get better results than the original algorithm.
\end{abstract}

\section{Introduction}

In M. Sefrioui and J. Periaux article Nash Genetic Algorithms: examples and applications proposed Nash GAs, in solving the multi-objective optimization problem, only once get a Pareto optimal solution, instead of Pareto optimal solution front surface, this is obviously not enough.

Based on the Nash GAs in the foundation, the union NSGA algorithm, the paper proposes a kind of can get more Pareto optimum solution of the multi-objective optimization algorithm, Nash GAs with the combination of the NSGA algorithm, at the same time also played the role of accelerated NSGA algorithm convergence.

\section{The basic idea of the algorithm}

Algorithm can optimize object of every target, corresponding to the game of a participant, and all the independent variables in these between participators distribution, in which each participant distribution is a subset of the independent variables. In the optimization process, each player at the same time the use of genetic algorithm operation must be algebra optimize their corresponding target, until all goals are unable to optimize so far.

Algorithm will Nash equilibrium method embedded NSGA, in NSGA generation evolution ended, remove preserved the Pareto optimum solution of the external set, the use of Nash equilibrium thought optimization, the method is as follows: first take out the optimal solution of i-th Pareto $\left(X_{i 1}, X_{i 2}, \ldots, x_{i n}\right)$, its distribution to the first objective function (participant), a set of corresponding argument is $X_{1}$, random extraction subset $X^{\prime}{ }_{1}$ of $X_{1}$ (shall meet the requirements when $\left|X_{1}\right|=1$ or 2, $\left|X_{1}^{\prime}\right|=1$; others $\left.\frac{\left|X_{1}\right|}{4} \leq\left|X_{1}^{\prime}\right| \leq \frac{\left|X_{1}\right|}{2}\right), X_{1} \backslash X_{1}^{\prime}$, will set the argument with the first i-th Pareto optimal solutions corresponding value is fixed, the independent variable as a new objective 
function arguments. Will the new objective function, using the genetic algorithm optimization, this will be a single objective optimization problem, the use of SGA able to quickly solve, the final result will get a optimal solution, using the optimal solution update first Pareto. The optimal solution of the corresponding position of the value, will get a new Pareto optimal solution. Use the same method will be the Pareto optimal solution assigned to 2, 3,..., $k$ target function, and eventually add existing external set will get $M(k+1)$ solution ( $\mathrm{M}$ for the current external set scale). In these solution, eliminate the bad solution, if the number of final solution or less $\bar{N}$ ( $\bar{N}$ for external set scale), retains all the solution, as the current Pareto optimal solution set, will enter the next generation of population to cross and mutation operation: if the last solution quantity $\geqslant \bar{N}$, then use crowded distance criterion, retain crowded distance larger than before a individual as a new Pareto optimal solution set.

\section{Algorithm test and result analysis}

In order to facilitate comparison, selecting the following functions for test, that is to say, the problem is described as follows:

$$
\begin{aligned}
& \operatorname{Min}\left(f_{1}\left(x_{1}\right), f_{2}\left(x_{1}, x_{2}\right)\right) \\
& f_{1}\left(x_{1}\right)=4 x_{1} \\
& f_{2}\left(x_{1}, x_{2}\right)=g\left(x_{2}\right) \cdot h\left(f_{1}\left(x_{1}\right), g\left(x_{2}\right)\right) \\
& g\left(x_{2}\right)=\left\{\begin{array}{l}
4-3 \exp \left(-\left(\frac{x_{2}-0.2}{0.02}\right)^{2}\right) \text { if } 0 \leq x_{2} \leq 0.4 \\
4-2 \exp \left(-\left(\frac{x_{2}-0.7}{0.2}\right)^{2}\right) \text { if } 0.4 \leq x_{2} \leq 1
\end{array}\right. \\
& h\left(f_{1}, g\right)= \begin{cases}1-\left(\frac{f_{1}}{g}\right)^{a} & \text { if } f_{1} \leq g \\
0 & \text { otherwise }\end{cases} \\
& \alpha=0.25+3.75\left(g\left(x_{2}\right)-1\right) \quad x_{1} \in[0,1], x_{2} \in[0,1]
\end{aligned}
$$

This question has a concave local Pareto optimal solution set and a convex global optimal solution set this problem have a local not convex Pareto front end and a convex global Pareto front end.

Objective function were used respectively to linear weighted method, the NSGA and the proposed algorithm is presented to solve this problem, the results are as follows:

\section{The linear weighting method}

Using linear weighted method 50 times results:

$72 \%$ convergence in $\left(\begin{array}{l}x_{1}=0.235033 \\ x_{2}=0.200288\end{array}\right)$, the corresponding values of $f_{1}$ and $f_{2}$ are

$$
\left(\begin{array}{l}
f_{1}=0.940134 \\
f_{2}=0.0156209
\end{array}\right) \text {, }
$$

This point is located in global Pareto front end.

$28 \%$ convergence in $\left(\begin{array}{l}x_{1}=0.503219 \\ x_{2}=0.683019\end{array}\right)$, the corresponding values of $\mathrm{f}_{1}$ and $\mathrm{f}_{2}$ are

$$
\left(\begin{array}{l}
f_{1}=2.01288 \\
f_{2}=0.006432
\end{array}\right)
$$

This point is located in local Pareto front end.

The solution process using real number coding method, population scale is 20, the biggest evolution algebra is 2000 .

\section{NSGA}


Using real number coding, based on the Pareto sort and sharing mechanism of the NSGA solving this problem, population scale is 50 . Table 1 shows different evolutionary algebra, global Pareto optimal solution for the proportion of all solutions.

Table 1. The NSGA algorithm to solve the results

\begin{tabular}{|l|l|l|l|l|l|}
\hline Evolution algebra & 40 & 100 & 200 & 300 & 400 \\
\hline The proportion of global Pareto optimal solution & $60 \%$ & $60 \%$ & $90 \%$ & $90 \%$ & $100 \%$ \\
\hline
\end{tabular}

From the above we can see that when the population scale is 50, NSGA need to run 400 generation evolution to guarantee $100 \%$ convergence to the optimal solution.

\section{The algorithm of this paper}

In this paper two goals, two independent variables function optimization problem, we will first objective function as the first player, optimize shut; The second objective function as the second player, fixed gas, optimization of the people. In order to facilitate comparison, the population scale as 50, separately using linear weighting method, NSGA and the proposed algorithm is presented to solve the problem. Get the result is as follows:

Table 2. The comparison of the three kinds of algorithm

\begin{tabular}{|l|c|c|c|c|c|}
\hline Evolution algebra & 40 & 100 & 200 & 300 & 400 \\
\hline The proportion of weighted Pareto optimal solution & $26 \%$ & $32 \%$ & $35 \%$ & $35 \%$ & $54 \%$ \\
\hline The proportion of NSGA Pareto optimal solution & $60 \%$ & $60 \%$ & $90 \%$ & $90 \%$ & $100 \%$ \\
\hline The proportion of this paper algorithm Pareto optimal solution & $62 \%$ & $70 \%$ & $100 \%$ & $100 \%$ & $100 \%$ \\
\hline
\end{tabular}

Visibly, the proposed algorithm, the convergence speed of the optimal, only need 200 generation that can be $100 \%$ convergence to global Pareto optimal solution. The NSGA embedded in the process of the Nash equilibrium, so the algorithm of time is NSGA doesn't improve.

\section{Conclusions}

Based on the Nash equilibrium in the foundation, the union NSGA algorithm, and put forward a kind of solving multi-objective optimization problem of the genetic algorithm. Algorithm through the use of Nash equilibrium thoughts in the NSGA have obtained Pareto optimal solution based on the fixed part of the argument, optimize the other independent variable, continuously optimize Pareto optimal solution method, to speed up the convergence of the algorithm. Experiments show that the proposed algorithm has fast convergence characteristics, but the running time of the algorithm is NSGA and without too much improved, this is also put forward in this paper to improve the algorithm of a direction.

\section{References}

[1] V.Cours Pareto.D’ Eeonomies Politique, volume I and II [M].Lausanne: Rouge, 1896:34-36.

[2] F.Hausdorff, Mengenlehre. SetTheory [M].NewYork:ChelSea Publishing ComPany, 1957: 327329.

[3] J.Von Neumann, O.Morgenstern. The Theory of Games and Economic Behavior[M]. New Jersey: Princeton University Press,1944:89-90.

[4] H.W.Kuhn, A.W.Tueker. Nonlinear Programming, in " Proceeding of the Second Berley symposium on mathematical Statistics and Probabiliyt”. California: University of California Press, 1951:481-492.

[5] Fogel D B. Asymptotic Convergence Properties of Genetic Algorithms and Evolutionary Programming : Analysis and Experiments[J] .Cybernetics and System ,1994,25 (6) :389 - 407.

[6] Gambardella L M , Dorigo M. Solving symmetric and asymmetric TSPs by ant colonies[A]. In Proceedings of t he IEEE international conference on evolutionary computation ( ICEC '96) [C]. USA : IEEE Press , 1996. 622 - 627.

[7] Sobajic DJ, Pao YH. Artificial neural net based dynamic security assessment for electric power systems.IEEE Trans.On Power Systems, Vol.4(2009), p.220 226.

[8] Gordon R.T. Artificial neural network approach to assessment. Intelligent Engineering Systems Through Networks, Vol.4(2007), p.1175 1180. 
[9] Molina AM Chou K C. Application of Neural Networks for the Performance Evaluation of Bridges. Probabilistic Mechanics and Structural and Geotechnical Reliability, Proceedings of the Specialty Conference. Sponsored by ASCE,2008, p.298 301.

[10] M. R. Azimi-Sadjadi, R. J. Lion. Fast Learning Process of Multilayer Neural Networks Using Resursive Least Squares Method. IEEE Trans.On SP, Vol.40(2010), p.446 450.

[11]Yanmei Li, Jingmin Wang. Risk Assessment Model of Smart Grid Project Based on Variable P recision RS and LSSVM. Advances in Information Sciences and Service Sciences (AISS). Vol.3, N o.10, 2011,11 :375 383. 